of a series of these animals at different times before and after suture, he was able to follow the expansion and contraction of the columns as first one eye and then the other took control.

That process is not controversial, and has actually been photographed by D. Hubel (Harvard University) who showed some amazing slides of coronal sections of monkey visual cortex stained by the injection of labelled amino acid into one eye. The zebra stripes formed across the cortex by the ocular dominance columns were caught in the act of reciprocal contraction and expansion as a result of suture reversal, leaving no room for doubt about what was going on.

The disagreement arises over orientation selectivity, which Movshon and his associates claim is influenced by visual experience and Hubel and Wiesel claim is innately specified. Movshon's evidence comes from recordings of a few cells in reverse sutured kittens that could be stimulated from either eye. Such binocular cells in normal cats invariably have the same orientation selectivity for stimuli from right and left eyes. In the reverse sutured kittens, however, Movshon found a few binocular cells, presúmably in the process of changing their ocular allegiance, which seemed at the same time to be changing their orientation preference. These cells were selective for different orientations when stimulated through the different eyes.

While that would seem to be compelling evidence for plasticity under the positive influence of experience in the cat, Hubel has begun to test the effects of stripe-rearing (in which visual experience is limited to bars of a single orientation) in monkeys and (in the one satisfactory specimen so far) has found no effect on the columnar pattern of orientation selectivity of cortical cells. Challenged by Movshon, he could offer no explanation for the discrepancies; nor could Movshon explain recent failures to replicate striperearing results in cats.

\section{Perceptual problems}

Another embarrassment for visual neurophysiologists emerges from psychophysical experiments on stereopsis described by J. P. Frisby (University of Sheffield). According to the neurophysiology, stereopsis is mediated by binocular, orientation-selective cells which have a marked maximum response where the stimuli in either eye have a specific horizontal disparity on the retina. Frisby has tested depth perception in man (two men, himself and one other) using an adaptation of the Julesz random dot stereogram made with lines instead of dots. He has found that the perceived depth is a function of the length of the lines

\title{
Assessing prospects for Spacelab
}

\section{from John Gribbin}

A FEW months ago a series of experiments were flown on board NASA's CV-990 'flying laboratory' to simulate an orbital mission by the European Spacelab which is to be flown on the Space Shuttle during the 1980 s. Some of the first scientific results from this ASSESS mission are presented in a series of papers commencing on page 649 of this issue, but the other lessons learned are also of great interest.

The joint ASSESS mission illustrated that a low-cost programme with a low level of preparatory requirements, testing and documentation can operate successfully under the proper management. Not only was the ASSESS mission itself fairly cheap (by space agency standards); it provided every indication that low-cost projects can work well when the real Spacelab gets off the ground, which must be encouraging news for many of the research groups working within tight budgets to try to take advantage of the opportunities which will be provided by the next generation of manned spacecraft.

And such aircraft simulations may have a significant role to play in developing instrumentation for the next generation of unmanned spacecraft as well. The ASSESS flights included an experiment from the Laboratory for Atmospheric and Space Physics (LASP) of the University of Colorado, a scanning two-channel spectrometer

used, a result which defies explanation within the current physiological paradigm.

No satisfactory physiological paradigm has in any case yet been proposed for perceptual phenomena which depend on activity beyond the primary visual cortex, a point implicit in the title to which Stuart Sutherland (University of Sussex) addressed himself: "There's more to vision than meets the eye." Sutherland believes in the use of computer programs as a testable way of representing how feats such as human visual perception might be achieved, and the first lesson to be learned from such programs is the enormous amount of knowledge and past experience people bring to bear on seeing things (consider the difference between a layman and a trained histologist, looking at a microscope slide). The lesson from the current lumbering knowledgebased computer vision programs however seems to be that brains have some way of leaping to global conclusions while computers are still creeping along line by line. which covered the wavelength range $270-650 \mathrm{~nm}$ and was a prototype of the ultraviolet spectrometer which LASP expects to fly on the forthcoming Pioneer Venus orbiter spacecraft. The mission provided a far better opportunity to sort out bugs in the system than any ground based operation, as well as producing valuable observations of Venus.

But ASSESS proved that there will be real problems in the practical operation of a flying laboratory. Human engineering of instrument control is necessary, as is a near real time high quality visual display of recorded data to confirm to the operator that successful measurements are being made. One clear result emerged from the human side of ASSESS. The best results come from operators who are trained scientists, understand the equipment and can use a soldering iron effectively on the spot to overcome the troubles they have diagnosed. There will, it seems, be no retreaded test pilots on the Spacelab, only a professional astronaut-pilot and the professional scientistoperators.

A second mission is now planned. NASA has indicated that the CV-990 will be available in October/November 1976, which would allow time for the data collected to be usefully incorporated into the final stages of the development of Spacelab.

\section{Protoplasts at Nottingham}

from E. C. Cocking

The 4th International Meeting on Yeast and Other Protoplasts was held at Nottingham, UK on September $8-12$. The proceedings will be published by Academic Press.

THIS was one of the rare occasions when workers on bacterial, fungal and higher plant protoplasts came together to exchange viewpoints. As a result it was possible to compare the properties of protoplasts from both eukaryotic and prokaryotic systems; and for many of the participants this was the major attraction.

Enzymatic proceedings for the degradation of the cell wall, resulting in protoplast release, are now largely standard procedures; and, although the enzymes used for isolation differ in the various systems, the influence of the exact nature of the cell wall was 